\title{
Harmonic Quantum Coherence of Multiple Excitons in PbS/CdS Core-Shell Nanocrystals
}

\author{
Hirokazu Tahara, Masanori Sakamoto, Toshiharu Teranishi, and Yoshihiko Kanemitsu \\ Institute for Chemical Research, Kyoto University, Uji, Kyoto 611-0011, Japan \\ (Received 22 May 2017; published 11 December 2017)
}

\begin{abstract}
The generation and recombination dynamics of multiple excitons in nanocrystals (NCs) have attracted much attention from the viewpoints of fundamental physics and device applications. However, the quantum coherence of multiple exciton states in NCs still remains unclear due to a lack of experimental support. Here, we report the first observation of harmonic dipole oscillations in $\mathrm{PbS} / \mathrm{CdS}$ core-shell NCs using a phase-locked interference detection method for transient absorption. From the ultrafast coherent dynamics and excitation-photon-fluence dependence of the oscillations, we found that multiple excitons cause the harmonic dipole oscillations with $\omega, 2 \omega$, and $3 \omega$ oscillations, even though the excitation pulse energy is set to the exciton resonance frequency, $\omega$. This observation is closely related to the quantum coherence of multiple exciton states in NCs, providing important insights into multiple exciton generation mechanisms.
\end{abstract}

DOI: 10.1103/PhysRevLett.119.247401

Over the past three decades, semiconductor nanocrystals (NCs) have been extensively studied to gain fundamental knowledge of quantum confined excitons and their photonic applications. Their energy levels and electronic wave functions are controlled by the NC size and its heterostructure design $[1,2]$. NCs exhibit unique quantum characteristics, including multiple exciton generation (MEG) [3-7], strong Auger recombination of excitons [8,9], and long excitonic coherence [10-12]. In particular, the highly efficient photoluminescence at room temperature implies that both impurities and defects hardly exist in NCs $[13,14]$. A detailed understanding of generation and relaxation processes of multiple excitons is necessary to clarify these unique properties.

The generation and recombination dynamics of multiple excitons in NCs have been investigated by monitoring the number of photogenerated electrons and holes. For example, the lifetimes of multiple excitons are well explained by the quantized Auger recombination pathways of multiple excitons $[9,15]$. In contrast, more detailed discussions beyond a discrete change in the number of excitons, e.g., coherent states of multiple excitons, are important for a deep understanding of the nature of multiple excitons. Theoretical investigations of MEG have predicted that one high-energy exciton generates multiple low-energy excitons via a coherent superposition between the single and multiple exciton states [16,17]. The MEG is anticipated for use in photonic applications such as highly efficient solar cells [18-22] and ultraviolet photodetectors [23]. Therefore, the observation of

Published by the American Physical Society under the terms of the Creative Commons Attribution 4.0 International license. Further distribution of this work must maintain attribution to the author(s) and the published article's title, journal citation, and DOI. the theoretically predicted coherent state is essential for discussions on MEG mechanisms and the dynamical nature of multiple excitons in NCs. However, due to a lack of experimental support, it remains unclear whether multiple excitons generate their coherent states in the NCs or not. Coherence of multiple excitons has not been clarified thus far by the conventional approach to the forward process of MEG, i.e., generation of multiple excitons from a highenergy state. This problem can be solved elegantly by clarifying the physics of the reverse process of MEG, i.e., generation of a high-frequency state from multiple excitons. The observation of the reverse process provides a fundamental key for understanding the details of MEG, equally important as the investigation of the forward process. Recently, phase-sensitive spectroscopies allow for sophisticated measurements of the multiple electronic states [24-28]. NCs have also been examined by using them to understand the dynamics of multiple excitons $[29,30]$. Thus, these phasemonitoring techniques enable us to analyze coherent dipole oscillations.

Here, we report the observation of coherent multiple excitons in $\mathrm{PbS} / \mathrm{CdS}$ core-shell NCs with a spectroscopic technique using phase-locking of excitation pulses. We demonstrate that the phase-locked interference detection of the transient absorption (TA) clearly reflects the frequency-multiplied dipole oscillations in the NCs. From the coherent dynamics and the excitation-photon-fluence dependence of the oscillations, we found that the oscillations are caused by multiple excitons. This harmonic quantum coherence composed of multiple excitons provides significant information for manipulation of multiple excitons states.

The experimental setup for phase-locked interference detection of TA signals is illustrated in Fig. 1(a). In the experiment, a stable phase-locked pulse pair was produced by a phase-locking feedback system (see Fig. S1 in the Supplemental Material [31]) and used as pump pulses with 

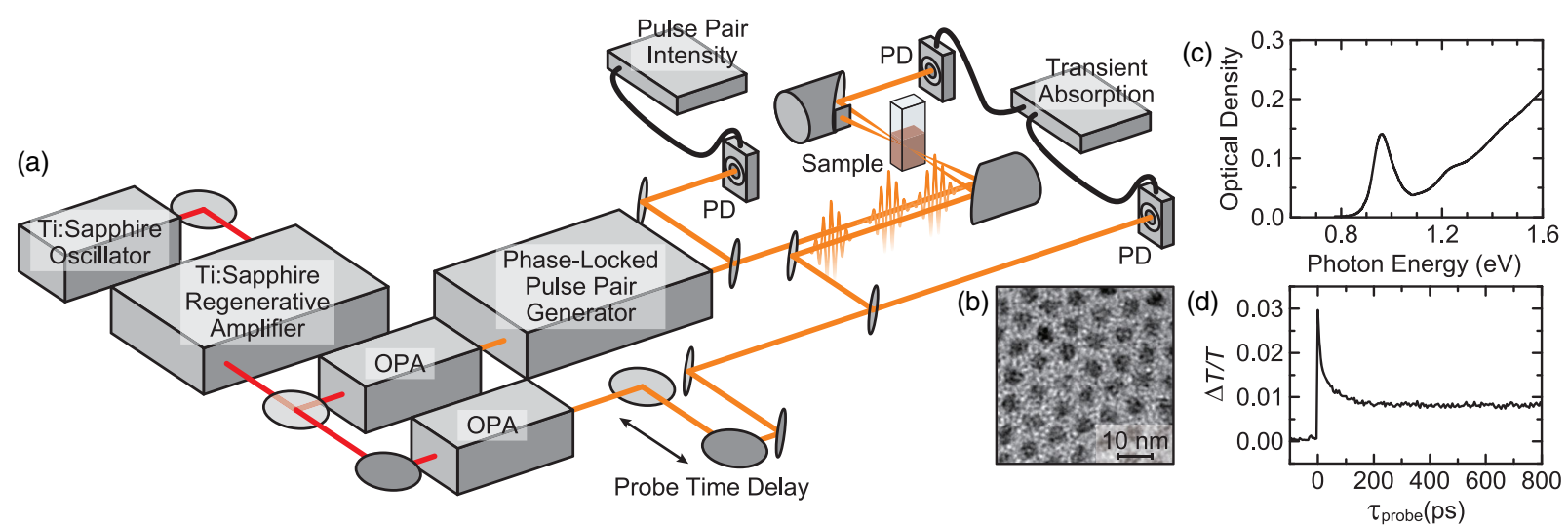

FIG. 1. (a) Schematic of setup for phase-locked interference detection of transient absorption. OPA: optical parametric amplifier; PD: photodetector. (b) Transmission electron microscope image and (c) absorption spectrum of $\mathrm{PbS} / \mathrm{CdS}$ core-shell nanocrystals. (d) Transient absorption signal for the probe time of -100 to $800 \mathrm{ps}$.

variable time intervals between the pump-pulse pair. The TA signal and the interference intensity of the pump-pulse pair were measured simultaneously as a function of the time interval between the pulses. In addition to the first and second pump pulses, a third pulse was used as a probe pulse to measure the TA signals. The sample used in this study was an ensemble of $\mathrm{PbS} / \mathrm{CdS}$ core-shell NCs in toluene solution. $\mathrm{PbS}$ NCs are one of the most suitable systems for this experiment to observe multiple excitons since they are expected to provide high-density electronic states as a result of the high degeneracies of their lowest states [32]. The details of the synthesis process $[33,34]$ are described in the Supplemental Material [31]. The solution was stirred to remove the effect of photoinduced charging [35]. The transmission electron microscope image and the absorption spectrum of the sample are shown in Figs. 1(b) and 1(c), respectively. The microscope image shows that the NCs have been synthesized with a fairly homogeneous size distribution. In the absorption spectrum, we observed the exciton resonance at $0.96 \mathrm{eV}$. The photon energies of the pump and probe pulses were tuned to this exciton resonance. Figure 1(d) shows the TA signal from a standard pump-probe measurement, i.e., using a single-pulse pump. The rapid decay within $200 \mathrm{ps}$ is assigned to Auger recombination of multiple excitons [8,9], while the long-lifetime component across the entire measurement time range reflects the recombination of single excitons [36]. To reveal the coherent dynamics of multiple excitons, it was required to study the initial TA dynamics with the phase-locked interference detection.

Figure 2(a) shows the experimental result with the phaselocked interference detection. In this measurement, the total time interval between the pump-pulse pair is equal to $\tau_{\text {pump }}+\Delta \tau_{\text {pump }}$. The vertical time axis $\tau_{\text {pump }}$ corresponds to the macro time step, which was controlled via a steppermotor-driven delay stage with a delay increment of $20 \mathrm{fs}$. The horizontal time axis $\Delta \tau_{\text {pump }}$ corresponds to the subwavelength-scale change in the optical path length of the second pump pulse, which was controlled by a piezoelectric actuator allowing for a time resolution of $0.53 \mathrm{fs}$. We measured excitonic dipole oscillations by scanning the micro time $\Delta \tau_{\text {pump }}$ for each macro time step of $\tau_{\text {pump }}$. Therefore, $\tau_{\text {pump }}$ allows us to scan the envelope coherent dynamics of the excitonic dipole oscillations, and $\Delta \tau_{\text {pump }}$ provides exact information about the frequencies of the oscillations. To obtain strong TA signals for all delay times, the probe time was set at 140 fs. To clarify the line shape of the oscillating behavior, the signal for $\tau_{\text {pump }}=0 \mathrm{fs}$ is

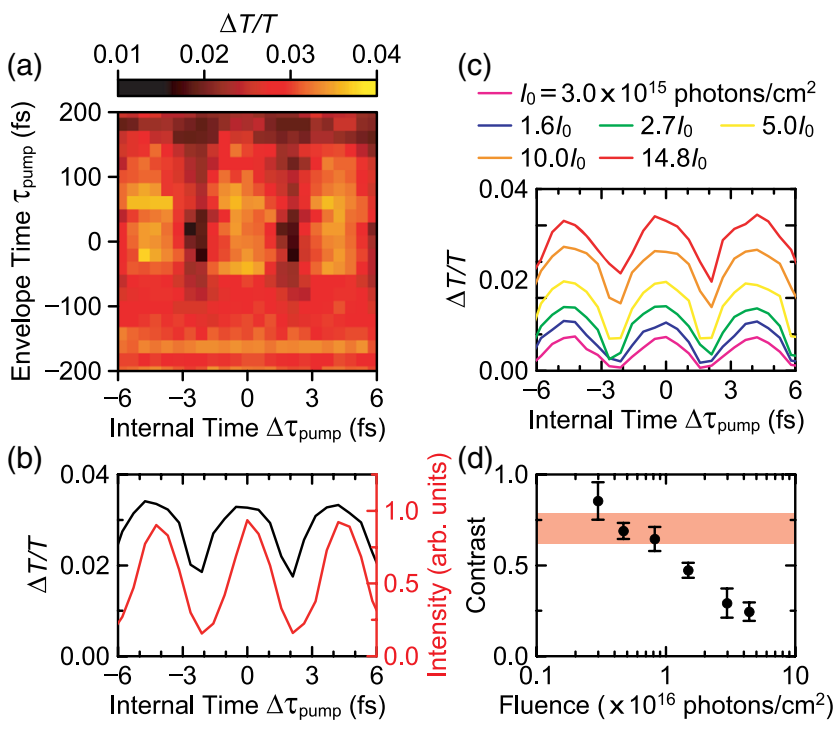

FIG. 2. (a) Transient absorption signal as a function of internal and envelope times. (b) Transient absorption signal and interference intensity of the pump-pulse pair at $\tau_{\text {pump }}=0 \mathrm{fs}$. (c) Excitation-photon-fluence dependence of transient absorption signals at $\tau_{\text {pump }}=0$ fs. (d) Excitation-photon-fluence dependence of the contrast calculated from the oscillating transient absorption signals. The shaded area corresponds to the contrast range expected for signals that are dominated by absorption nonlinearity. 
plotted in Fig. 2(b) with the black curve. For comparison, the interference intensity of the pump-pulse pair is also plotted with the red curve. The pump-pulse pair interference clearly shows a sinusoidal oscillation with a frequency corresponding to the wavelength of the pump laser. In contrast, the TA signal shows an oscillation that cannot be expressed with a single sinusoidal oscillation. This nonsinusoidal oscillation reflects the ultrafast response of photoexcited electronic states. To explain this nonsinusoidal oscillation, we consider two origins: (1) absorption nonlinearity and (2) superposition of several coherent states with different frequencies.

To determine the origin of the nonsinusoidal oscillation, we measured the excitation-photon-fluence dependence of the oscillating signals as shown in Fig. 2(c). The TA signal shows a sinusoidal oscillation for the weakest excitation, while it becomes more nonsinusoidal with increasing excitation-photon fluence. To investigate a possible explanation with absorption nonlinearity, we have to examine whether the measured maxima $\left(\Delta T / T_{\max }\right)$ and minima $\left(\Delta T / T_{\min }\right)$ of all curves lie within a certain range determined by absorption nonlinearity or not. Therefore, we consider the range determined by $\Delta T / T \propto I^{\gamma}$ with $\alpha \leq \gamma \leq 1$. In the highest excitation intensity regime, our sample showed a maximum nonlinearity of $\alpha=0.68$. Since the ratio of the excitation intensity maxima $\left(I_{\max }\right)$ and minima $\left(I_{\min }\right)$ is fixed at all powers $\left(I_{\min }=\beta I_{\max }\right)$, the ratios of the maxima and minima of the measured $\Delta T / T$ must obey the same power law in case of a dominant absorption nonlinearity. However, if the experimental results do not obey this power law, the absorption nonlinearity can be ruled out for the origin of the nonsinusoidal oscillation. The range, determined by $\alpha \leq \gamma \leq 1$ and where the measured points have to lie within, can be conveniently visualized with the so-called contrast, defined by $\left(\Delta T / T_{\max }-\Delta T / T_{\min }\right) /\left(\Delta T / T_{\max }+\Delta T / T_{\min }\right)$. Using the absorption nonlinearity $\Delta T / T \propto I^{\gamma}$, we obtain a contrast range $\left(1-\beta^{\gamma}\right) /\left(1+\beta^{\gamma}\right)=0.62$ to 0.79 for $\alpha \leq \gamma \leq 1$ (in our experiment $\beta=0.12$ ). This range and the contrast of the oscillating transient absorption signals are plotted as a function of the excitation-photon fluence as shown in Fig. 2(d). The contrast of the measured data decreases with increasing excitation-photon fluence. Since the experimental results decrease far beyond the power law area, we conclude that the experimental results cannot be explained by a simple nonlinear absorption.

Next, we examined the contribution of superpositions of several coherent states in the oscillating signals. To analyze the nonsinusoidal oscillations, we discuss the two-pulsepump excitation sequences of electronic states via doublesided Feynman diagrams [37,38]. By taking the excitation sequences up to third-order oscillation into account, the TA signal can be decomposed into $n \omega$ oscillations, where $n$ is the harmonic order $(n=1,2,3)$ and $\omega$ corresponds to the exciton resonance frequency. The major excitation

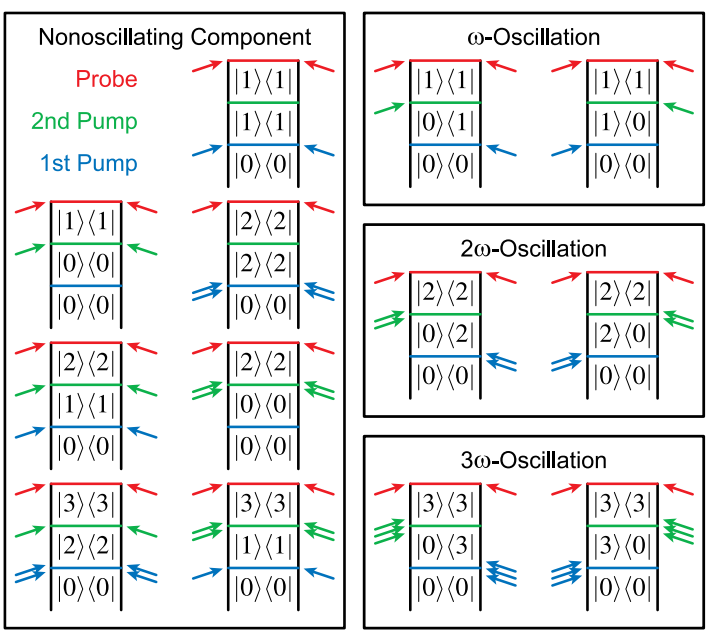

FIG. 3. Double-sided Feynman diagrams for two-pulse-pump transient absorption. The state generated by $n$-photon absorption is denoted by $|n\rangle(n=1,2,3)$.

sequences are shown in Fig. 3, where the state generated by $n$-photon absorption is denoted by $|n\rangle$. Higher order contributions to the TA are shown in Fig. S3 in the Supplemental Material [31]. Here, the $|n\rangle$-state corresponds to multiple excitons in the ground state rather than one highenergy exciton generated by carrier upconversion. The upconversion via the biexciton Auger recombination was not observed in our phase-locked measurement since the biexciton Auger recombination lifetime of $\sim 60 \mathrm{ps}$ is much longer than the probe time of $140 \mathrm{fs}$. Furthermore, the excitation-photon-fluence dependence described below cannot be explained with upconversion processes [31]. By using the fitting function $\Delta T / T=A_{0}+A_{1} \cos \left(\omega \Delta \tau_{\text {pump }}\right)-$ $A_{2} \cos \left(2 \omega \Delta \tau_{\text {pump }}\right)+A_{3} \cos \left(3 \omega \Delta \tau_{\text {pump }}\right)$ [31], the amplitudes of the $n$ th-order oscillation $A_{n}$ in Fig. 2(b) are determined to be $A_{0}=2.8 \times 10^{-2}, A_{1}=7.0 \times 10^{-3}, A_{2}=2.4 \times 10^{-3}$, and $A_{3}=1.1 \times 10^{-3}$. The $2 \omega$ and $3 \omega$ oscillations cannot be explained by conventional coherence with the energy difference corresponding to $\omega$. Therefore, transient dipoles between highly separated states in the excitation diagrams are required for the high-frequency oscillations. We hypothesize that the efficient generation of the harmonic quantum coherences correlates with multiple excitons in NCs.

To reveal the correlation of the above-mentioned harmonics with the multiple excitons, we analyzed the excitationphoton-fluence dependence of the harmonic amplitudes. The harmonic amplitudes are plotted in Fig. 4(a), showing an initial increase in the low photon-fluence regime and saturation for high photon-fluence regime of more than $1.0 \times 10^{16}$ photons $/ \mathrm{cm}^{2}$. This saturation behavior is similar to that predicted by the Poisson distribution of multiple excitons $[9,36]$. As shown in the double-sided Feynman diagrams in Fig. 3 (see also Fig. S3 in the Supplemental Material [31]), the absorption of a number of photons equal to or larger than $n$ is required to generate the $n \omega$ oscillation. The 


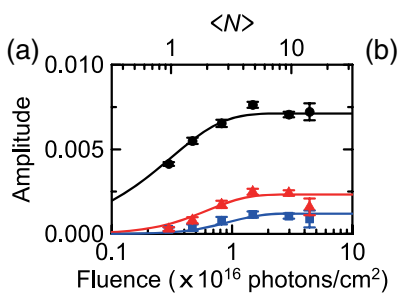

(b)

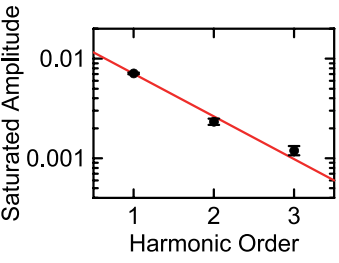

(c)

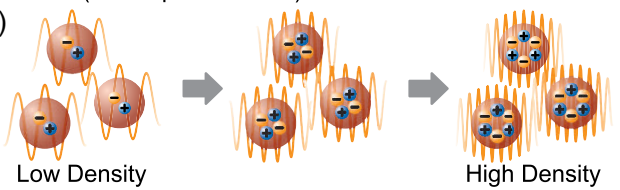

FIG. 4. (a) Amplitudes of harmonic quantum coherences. Amplitudes of the first-, second-, and third-order oscillations are plotted as closed circles, triangles, and squares, respectively. The average number of absorbed photons per nanocrystal is denoted by $\langle N\rangle$. The calculated generation probabilities of the oscillations are shown with the solid curves. (b) Saturated amplitudes of the harmonic quantum coherences. (c) Schematic of coherent dipole oscillations with increasing exciton density.

formation probability of the $n \omega$ oscillation can be therefore expressed by the generation of multiple excitons according to the Poisson distribution of absorbed photons. We assume that the $n \omega$ oscillation is generated by all processes with an absorbed photon number equal to or larger than $n$, which is determined by a function including the Poisson distribution [31]. Then, we calculated the generation probabilities of $\omega$, $2 \omega$, and $3 \omega$ oscillations as shown in Fig. 4(a) by the solid curves. We fitted the experimental data with an absorption cross section of $\sigma=3.2 \times 10^{-16} \mathrm{~cm}^{2}$ [31]. Figure 4(a) shows that the experimental TA amplitudes correlate well with the predicted generation probabilities of the harmonics. Since the generation probabilities are calculated from the Poisson distribution of multiple excitons, the good correlation in Fig. 4(a) evidences that the harmonic quantum coherences are generated by multiple excitons.

The harmonic amplitudes reach the saturation region under the high excitation densities as shown in Fig. 4(a). To understand the saturated ratio of the harmonic quantum coherences, the saturated amplitudes of $\omega, 2 \omega$, and $3 \omega$ oscillations are plotted in Fig. 4(b). The negative power law dependence on the harmonic order in Fig. 4(b) corresponds to the decreasing tendency of the multiphoton absorption $[39,40]$. This result explains the mechanism of harmonic quantum coherence in NCs as shown schematically in Fig. 4(c). Under low excitation conditions, only single excitons are generated and show a coherence with $\omega$ oscillation. As the excitation-photon fluence increases, the exciton density becomes high enough to generate multiple excitons. In our experiment, multiple excitons are directly generated by multiphoton absorption under the resonant excitation of the ground-state excitons. Thus, we do not consider the formation time of multiple excitons or the exciton-exciton interactions $[41,42]$. Multiple excitons are occupied in such a way that the energy conservation is
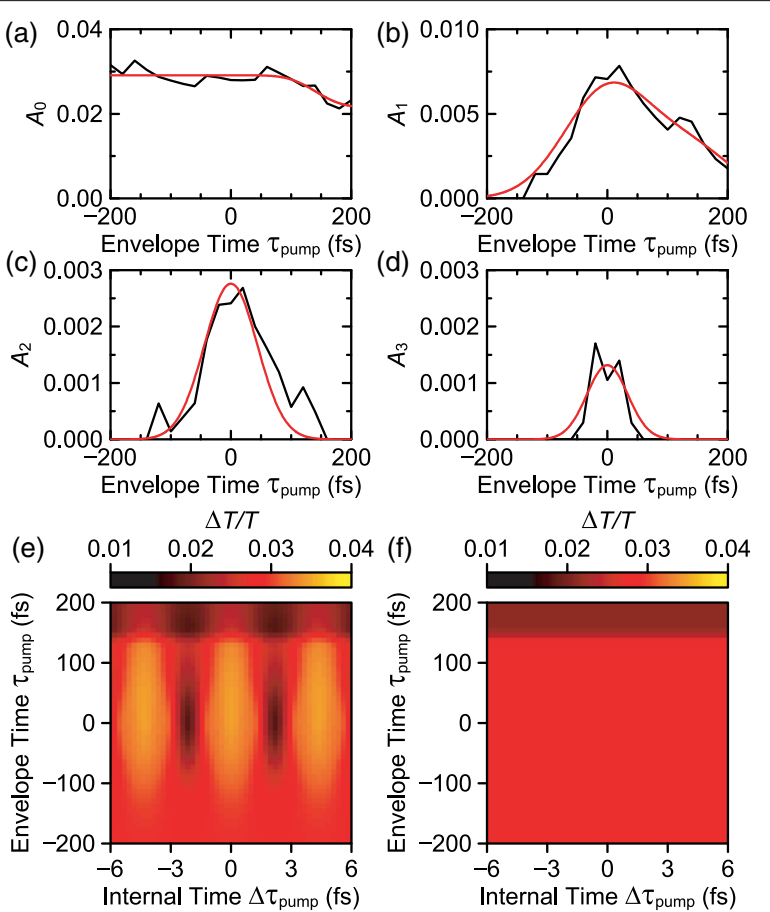

$\Delta T / T$

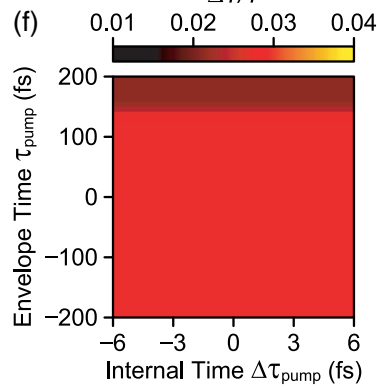

FIG. 5. Transient absorption amplitudes of (a) nonoscillating, and (b) first-, (c) second-, and (d) third-order oscillating components. Theoretical transient absorption signals under (e) coherent and (f) incoherent conditions.

satisfied. Therefore, high-frequency dipole oscillations, i.e., $2 \omega$ and $3 \omega$ oscillations, are caused by the direct formation of the transient dipoles between the multiple exciton states and the ground state. This sequence clarifies that multiple excitons cause collective coherences with $2 \omega$ and $3 \omega$ oscillations, instead of a coherent system with $\omega$ oscillation for each exciton.

Ultrafast decoherence processes of the harmonic quantum coherences are also determined by the transient dipole oscillations of multiple excitons. To understand the coherent dynamics, we analyze the envelope dynamics of the dipole oscillations as shown in Figs. 5(a)-5(d). The decay times of the dipole oscillations become shorter for higher harmonic orders. This behavior is also the evidence for frequency multiplication in NCs since the free-induction decays are accelerated with increasing the dipole-oscillation frequency. Free-induction decays of transient dipole oscillations are known to originate from inhomogeneous broadening of the resonance energy [43]. The NC ensemble includes a crystal-size distribution that causes inhomogeneous broadening of the resonance energy. Regarding the nonoscillating component $A_{0}$, a decrease is observed at the times longer than $100 \mathrm{fs}$, which corresponds to the crossover region from two-pulse to one-pulse pump conditions. The envelope times being longer than the probe time, $140 \mathrm{fs}$, means that the probe pulse arrives before the second pump pulse. Therefore, the nonoscillating component for long envelope times results in the one-pulse-pump TA 
signals. Taking the free-induction decays of the harmonics and the crossover region into account, we calculated the TA amplitudes as shown in Figs. 5(a)-5(d) by the red curves [31]. The calculated results are in good agreement with the experimental results, which suggests that the harmonic dipole oscillations are determined by the harmonic freeinduction decays. Finally, we show the theoretical twopulse-pump TA signals under coherent (phase-locked pulses) and incoherent (random phase) conditions in Figs. 5(e) and 5(f), respectively. The coherent signal [Fig. 5(e)] reproduces the experimental results shown in Fig. 2(a) well, while the incoherent signal [Fig. 5(f)] is much flatter than that shown in Fig. 2(a). This means that phase locking of the excitation pulses is required to observe the harmonic quantum coherences. In contrast, for negative delays longer than $-150 \mathrm{fs}$, the coherent signal coincides with the incoherent signal. Therefore, the envelope dynamics show a transition from coherent to incoherent multiple excitons.

In conclusion, we observed harmonic quantum coherence of multiple excitons in semiconductor NCs for the first time using a phase-locked interference detection of TA. We confirmed that the observed harmonics with $\omega, 2 \omega$, and $3 \omega$ oscillations are generated by coherent multiple excitons. This means that a high-frequency collective coherence is formed in NCs. Since these high-frequency oscillations are closely related to high-energy excitons, they can strongly enhance MEG: one hot exciton with an energy corresponding to $3 \omega$ frequency is expected to generate three lowenergy excitons via $3 \omega$ dipole oscillation. The observed harmonic coherences can work as intermediate states that assist the MEG processes from a high-energy single exciton to multiple ground-state excitons. We believe that a deeper understanding of coherent multiple excitons will open a new pathway to enhanced carrier multiplication using their frequency-multiplied dipole oscillations.

Part of this work was supported by JST-CREST (Grant No. JPMJCR16N3) and JSPS KAKENHI (Grant No. 16H06520).

*Corresponding author.

tahara.hirokazu.7m@kyoto-u.ac.jp

${ }^{\dagger}$ Corresponding author.

kanemitu@scl.kyoto-u.ac.jp

[1] X. Peng, L. Manna, W. Yang, J. Wickham, E. Scher, A. Kadavanich, and A. P. Alivisatos, Nature (London) 404, 59 (2000).

[2] S. A. Ivanov, A. Piryatinski, J. Nanda, S. Tretiak, K. R. Zavadil, W. O. Wallace, D. Werder, and V. I. Klimov, J. Am. Chem. Soc. 129, 11708 (2007).

[3] R. D. Schaller and V. I. Klimov, Phys. Rev. Lett. 92, 186601 (2004).

[4] R. J. Ellingson, M. C. Beard, J. C. Johnson, P. Yu, O. I. Micic, A. J. Nozik, A. Shabaev, and A. L. Efros, Nano Lett. 5, 865 (2005).
[5] R. D. Schaller, M. Sykora, J. M. Pietryga, and V. I. Klimov, Nano Lett. 6, 424 (2006).

[6] A. Ueda, K. Matsuda, T. Tayagaki, and Y. Kanemitsu, Appl. Phys. Lett. 92, 233105 (2008).

[7] F. Hu, B. Lv, C. Yin, C. Zhang, X. Wang, B. Lounis, and M. Xiao, Phys. Rev. Lett. 116, 106404 (2016).

[8] V. I. Klimov, A. A. Mikhailovsky, D. W. McBranch, C. A. Leatherdale, and M. G. Bawendi, Science 287, 1011 (2000).

[9] V. I. Klimov, J. A. McGuire, R. D. Schaller, and V. I. Rupasov, Phys. Rev. B 77, 195324 (2008).

[10] P. Borri, W. Langbein, S. Schneider, U. Woggon, R. L. Sellin, D. Ouyang, and D. Bimberg, Phys. Rev. Lett. 87, 157401 (2001).

[11] H. Tahara, Y. Ogawa, F. Minami, K. Akahane, and M. Sasaki, Phys. Rev. B 87, 035304 (2013).

[12] H. Tahara, Y. Ogawa, F. Minami, K. Akahane, and M. Sasaki, Phys. Rev. Lett. 112, 147404 (2014).

[13] X. Brokmann, L. Coolen, M. Dahan, and J. P. Hermier, Phys. Rev. Lett. 93, 107403 (2004).

[14] T. Ihara and Y. Kanemitsu, Phys. Rev. B 92, 155311 (2015).

[15] Y. Kanemitsu, Acc. Chem. Res. 46, 1358 (2013).

[16] A. Shabaev, A. L. Efros, and A. J. Nozik, Nano Lett. 6, 2856 (2006).

[17] A. Shabaev, C. S. Hellberg, and A. L. Efros, Acc. Chem. Res. 46, 1242 (2013).

[18] O. E. Semonin, J. M. Luther, S. Choi, H.-Y. Chen, J. Gao, A. J. Nozik, and M. C. Beard, Science 334, 1530 (2011).

[19] M. C. Beard, J. M. Luther, O. E. Semonin, and A. J. Nozik, Acc. Chem. Res. 46, 1252 (2013).

[20] M. B. Smith and J. Michl, Chem. Rev. 110, 6891 (2010).

[21] J. Lee, P. Jadhav, P. D. Reusswig, S. R. Yost, N. J. Thompson, D. N. Congreve, E. Hontz, T. Van Voorhis, and M. A. Baldo, Acc. Chem. Res. 46, 1300 (2013).

[22] D. N. Congreve, J. Lee, N. J. Thompson, E. Hontz, S. R. Yost, P. D. Reusswig, M. E. Bahlke, S. Reineke, T. Van Voorhis, and M. A. Baldo, Science 340, 334 (2013).

[23] V. Sukhovatkin, S. Hinds, L. Brzozowski, and E. H. Sargent, Science 324, 1542 (2009).

[24] T. Zhang, C. N. Borca, X. Li, and S. T. Cundiff, Opt. Express 13, 7432 (2005).

[25] E. M. Grumstrup, S.-H. Shim, M. A. Montgomery, N. H. Damrauer, and M. T. Zanni, Opt. Express 15, 16681 (2007).

[26] K. W. Stone, K. Gundogdu, D. B. Turner, X. Li, S. T. Cundiff, and K. A. Nelson, Science 324, 1169 (2009).

[27] H. Tahara and Y. Kanemitsu, Phys. Rev. B 90, 245203 (2014).

[28] H. Tahara and Y. Kanemitsu, Appl. Phys. Express 9, 032403 (2016).

[29] D. B. Turner, Y. Hassan, and G. D. Scholes, Nano Lett. 12, 880 (2012).

[30] K. J. Karki, J. R. Widom, J. Seibt, I. Moody, M. C. Lonergan, T. Pullerits, and A. H. Marcus, Nat. Commun. 5, 5869 (2014).

[31] See Supplemental Material at http://link.aps.org/ supplemental/10.1103/PhysRevLett.119.247401 for details of phase-locked pulse pair generator, synthesis of $\mathrm{PbS} / \mathrm{CdS}$ core-shell nanocrystals, excitation sequences in two-pulsepump transient absorption measurement, multiple excitons and upconverted high-energy excitons, absorption cross section, theoretical analysis of generation probabilities of harmonic oscillations, and theory of decay dynamics of harmonic dipole oscillations. 
[32] I. Kang and F. W. Wise, J. Opt. Soc. Am. B 14, 1632 (1997).

[33] J. M. Pietryga, D. J. Werder, D. J. Williams, J. L. Casson, R. D. Schaller, V. I. Klimov, and J. A. Hollingsworth, J. Am. Chem. Soc. 130, 4879 (2008).

[34] H. Choi, J.-H. Ko, Y.-H. Kim, and S. Jeong, J. Am. Chem. Soc. 135, 5278 (2013).

[35] J. A. McGuire, J. Joo, J. M. Pietryga, R. D. Schaller, and V. I. Klimov, Acc. Chem. Res. 41, 1810 (2008).

[36] G. Nair, S. M. Geyer, L.-Y. Chang, and M. G. Bawendi, Phys. Rev. B 78, 125325 (2008).

[37] S. Mukamel, Principles of Nonlinear Optical Spectroscopy (Oxford University Press, New York, 1995).
[38] H. Tahara, Y. Ogawa, F. Minami, K. Akahane, and M. Sasaki, Phys. Rev. B 89, 195306 (2014).

[39] R. W. Boyd, Nonlinear Optics, 3rd ed. (Academic Press, San Diego, 2008).

[40] T. Tamaya, A. Ishikawa, T. Ogawa, and K. Tanaka, Phys. Rev. Lett. 116, 016601 (2016).

[41] W. M. Witzel, A. Shabaev, C. S. Hellberg, V. L. Jacobs, and A. L. Efros, Phys. Rev. Lett. 105, 137401 (2010).

[42] J. M. Shacklette and S. T. Cundiff, Phys. Rev. B 66, 045309 (2002).

[43] T. Yajima and Y. Taira, J. Phys. Soc. Jpn. 47, 1620 (1979). 